\title{
Oxidative stress in patients with refractory temporal lobe epilepsy and mesial temporal sclerosis: Possible association with major depressive disorder? is
}

\author{
Gerardo Maria de Araújo Filho ${ }^{\mathrm{a}, *}$, Denise Poltronieri Martins ${ }^{\mathrm{b}}$, Angélica Marta Lopes ${ }^{\mathrm{b}}$, Beatriz de Jesus Brait ${ }^{\mathrm{b}}$, \\ Ana Eliza Romano Furlan a , Camila Ive Ferreira Oliveira ${ }^{b}$, Lucia Helena Neves Marques ${ }^{\text {c }}$, \\ Dorotéia Rossi Silva Souza ${ }^{b}$, Eduardo Alves de Almeida ${ }^{d}$
}

a Department of Psychiatry and Medical Psychology, Faculdade de Medicina de São José do Rio Preto (FAMERP), São Paulo, Brazil

${ }^{\mathrm{b}}$ Department of Biochemistry and Molecular Biology-NPBIM, Faculdade de Medicina de São José do Rio Preto (FAMERP), São Paulo, Brazil

' Department of Neurological Sciences, Faculdade de Medicina de São José do Rio Preto (FAMERP), São Paulo, Brazil

d Department of Chemistry and Environmental Sciences - IBILCE, Universidade Estadual Paulista (UNESP), São Paulo, Brazil

\section{A R T I C L E I N F O}

\section{Article history:}

Received 29 September 2017

Revised 19 December 2017

Accepted 22 December 2017

Available online 3 February 2018

\section{Keywords:}

Temporal lobe epilepsy

Refractory epilepsy

Major depressive disorder

Oxidative stress markers

\begin{abstract}
A B S T R A C T
Objective: The objective was to evaluate the genetic and biochemical profiles associated with oxidative stress (OS) in patients with temporal lobe epilepsy with mesial temporal sclerosis (TLE-MTS) and a healthy control group, and also to verify the possible existence of association between OS markers and psychiatric disorders (PD) in group with TLE-MTS.

Methods: Forty-six patients with refractory TLE-MTS and 112 healthy controls were included. Psychiatric evaluation occurred through Diagnostical and Statistical Manual of Mental Disorders (DSM-5) criteria. A peripheral blood sample was collected for analysis of glutathione S-transferase (GST) T1/M1 polymorphisms and serum levels of malondialdehyde (MDA) and antioxidant capacity equivalent to the trolox (TEAC), serum markers of OS. Student's t-test, Fisher's exact test, Chi-square test, and Analysis of Variance (ANOVA) were used, with a significance level of $\mathrm{P}<0.05$.

Results: The PD were observed in 27 patients of the group with TLE-MTS (58.6\%); major depressive disorder (MDD) was the most frequent. Serum levels of MDA $(\mathrm{P}<0.0001)$ and TEAC $(\mathrm{P}<0.0001)$ were higher in group with TLE-MTS. When patients with MDD were compared with patients without PD, significant differences were observed between MDA $(P=0.002)$ and TEAC $(P=0.003)$ serum levels. Patients with TLE-MTS and MDD presented higher levels when compared with patients with TLE-MTS without PD and with another PD except MDD.

Conclusions: The present study observed significantly higher serum levels of MDA and of TEAC in patients with refractory TLE-MTS in comparison with the control group. The MDD was observed as an important issue associated with higher OS levels in refractory TLE-MTS. Further studies are needed to investigate the association of OS, TLE-MTS, and PD.
\end{abstract}

(C) 2017 Elsevier Inc. All rights reserved.

\section{Introduction}

Temporal lobe epilepsy (TLE) is the most frequent epilepsy syndrome, which may be associated with head trauma, cerebral malformations, infections, and febrile seizures [1]. Mesial temporal sclerosis (MTS) is the etiology most frequently associated with TLE (TLE-MTS), and is also the most frequent etiology observed in patients with epilepsy with seizures that are refractory to pharmacological

\footnotetext{
it The authors declare no conflicts of interest of any kind.

* Corresponding author at: Faculdade de Medicina de São José do Rio Preto (FAMERP), Av. Brigadeiro Faria Lima, 5416, 15090-000 São José do Rio Preto, SP, Brazil.

E-mail address: gerardo.filho@famerp.br (G.M. de Araújo Filho).
}

therapy [1-4]. Psychiatric disorders (PD), in turn, are among the most frequent and important aspects associated with TLE-MTS, causing several behavioral changes and making difficult the clinical and surgical management of these patients [5]. Due to the high frequency of PD in patients with TLE-MTS, there is a need for careful psychiatric evaluation especially for surgery candidates $[5,6]$.

Oxidative stress (OS), defined as the instability of free radical (FR) production, such as reactive oxygen species (ROS) and reactive nitrogen species (RNS) [7], is involved in many neurological diseases, including epilepsy $[1,8]$. The cells of the brain are sensitive to ROS, which can cause irreversible damage to biological molecules such as proteins, lipids, carbohydrates, and deoxyribonucleic acid (DNA), and can lead to cell death [9]. Glutathione S-transferases (GSTs) are a family of 
Phase II dimer proteins (conjugation reactions) that have a polymorphic character, with role in the detoxification of several ROS and RNS [10]. The OS processes activate GSTs, and their glutathione S-transferase M1 (GSTM1) and glutathione S-transferase T1 (GSTT1) variants act as catalysts in the detoxification of numerous ROS and RNS products [11].

The products of lipid peroxidation, such as the dosage of malondialdehyde (MDA) and the antioxidant capacity equivalent to the trolox (TEAC) in biological systems have been considered important parameters for evaluating cellular OS. The MDA has a cytotoxic and genotoxic actions, being found at high levels in some diseases associated with OS, while TEAC evaluates the antioxidant ability in eliminating ROS and RNS $[12,13]$. To date, there are few studies which have evaluated the levels of serum markers of OS in patients with TLE-MTS, as well as their association to GST polymorphisms, including GSTT1 and GSTM1. The objective of this controlled study was to verify the genetic and biochemical profiles in a series of patients with refractory TLE-MTS, considering the serum levels of MDA and TEAC, biomarkers of OS, and the genetic polymorphisms of GSTs.

\section{Methods}

\subsection{Subjects}

All patients were followed-up in the outpatient's clinic of a tertiary center (Epilepsy Section of the Faculdade de Medicina de São Jose do Rio Preto, São Paulo, Brazil), from February 2015 to July 2016. After local ethics committee approval and the informed consent has been assigned, 46 patients with refractory TLE-MTS were included in the study. Inclusion criteria were patients with age above 18 years old, presence of electroclinical diagnosis of TLE based on International League Against Epilepsy (ILAE) classification [14] and followed-up for at least 6 months in our unit. All patients also had clear magnetic resonance imaging (MRI) findings consistent with unilateral MTS and concordant interictal and ictal electroencephalogram (EEG) data. Exclusion criteria were clinical or other neurological illnesses besides epilepsy, cognitive impairments precluding psychiatric and clinical evaluations, and antihistamine administration or alcohol consumption within $72 \mathrm{~h}$ prior to the psychiatric evaluation. The control group consisted of 112 healthy individuals from the community. All participants answered a questionnaire to register clinical and sociodemographic data and collected a peripheral blood sample for genetic GSTT1/GSTM1 polymorphism analysis and measurements of MDA/TEAC serum levels.

\subsection{Procedures}

All patients with TLE-MTS underwent 2-6 days of continuous videoelectroencephalographic (VEEG) monitoring with 32-channel EEG recording, with electrodes placed according to $10-10$ system on the temporal lobe, including sphenoidal electrodes. The MTS was defined if atrophy, an increased T2-weighted signal, a decreased T1-weighted signal, and disrupted internal structure of the hippocampus were present accompanied by atrophy of the amygdala and/or temporal pole signal alteration on visual inspection of MRI pictures. The epileptogenic zone was determined by predominantly ipsilateral interictal epileptiform discharges (80\% cutoff) and seizure onset recorded during prolonged VEEG monitoring. The association between MTS side and the frequency of interictal epileptiform discharges on VEEG was also analyzed, and a cutoff of $>80 \%$ was considered for a predominant ipsilateral activity. Epilepsy was considered resistant to medical treatment when seizures persisted after the utilization of at least two first-line medications for partial seizures at highest tolerated doses. Initial precipitant injury (IPI) was defined as the occurrence of severe cerebral events in the first year of life before the appearance of epilepsy that required medical intervention and/or hospitalization. Febrile seizures, meningoencephalitis, head trauma, or severe perinatal hypoxia were considered as IPI.

\subsection{Psychiatric evaluation}

A single psychiatrist (GMAF) conducted the clinical interviews through the Diagnostical and Statistical Manual of Mental Disorders (DSM-5) criteria [15]. Since each patient could have had more than one axis I psychiatric diagnosis, the number of patients diagnosed and all comorbid PD diagnosed were both considered. The presence of other specific psychiatric diagnoses of epilepsy not covered by DSM-5 and well-described in literature, such as the interictal dysphoric disorder (IDD) and the psychoses of epilepsy were evaluated using the ILAE criteria, and such criteria were also utilized to differentiate postictal psychosis (PIP) from interictal psychosis (IIP) [16]. Data about lifetime history of psychiatric treatment, defined as any treatment with psychiatric drugs occurred in the past, were collected with patients in the first clinical interview. In addition, information about family history of epilepsy and PD were also obtained from patients through broad questions asking if any first-degree relative was in treatment for epilepsy and/or any PD at the moment of clinical interview.

\subsection{Biochemical evaluation}

To establish the genetic profile of the GSTM1 and GSTT1 variants, the genomic DNA was extracted from whole blood (5 mL), collected with ethylenediamine tetraacetic acid (EDTA), by the salting-out method carried out in three stages, comprising the following: 1) lysis of blood cells; 2) deproteinization; and 3) DNA precipitation and resuspension, followed by polymerase chain reaction (PCR) amplification $[17,18]$. These polymorphisms do not require enzymatic restriction after PCR, being identified by the presence or absence of alleles. Individuals were classified as having one or two copies of the gene (nonnull genotype), or lacking any copies of the gene (null genotype). The post-PCR product was separated by $1.5 \%$ agarose gel electrophoresis under a constant current of $150 \mathrm{~V}$ for $45 \mathrm{~min}$, separating fragments with 423 base pairs (bp) (GSTT1), 310 bp (control Cytochrome (CYP)), and 230 bp (GSTM1).

A method based on the reaction of MDA with thiobarbituric acid (TBA), which was detected by means of high performance liquid chromatography coupled to UV-vis/detector (HPLC-UV/VIS) at $535 \mathrm{~nm}[19,20]$. The TEAC was determined according to its equivalence to a known potent antioxidant, TROLOX (6-hydroxy-2,5,7,8tetramethrotonone-2-carboxylic acid; Aldrich Chemical Co., 23881-3), synthetic analogue of water-soluble vitamin $\mathrm{E}$, according to the method proposed by Miller et al. and modified by Re et al. The absorbance was measured at $734 \mathrm{~nm}$ using a Spectrophotometer (T60 Visible) $[21,22]$.

\subsection{Statistical analyses}

Student's $t$-test was applied for quantitative variables with mean and median values and Fisher's exact test or Chi-square test for qualitative variables. The Analysis of Variance (ANOVA) was applied for multiple comparisons, and Bonferroni adjustment for multiple tests was applied as a post hoc test for statistically significant differences. The GSTM1 and GSTT1 polymorphisms were analyzed according to the presence or absence of the independent and associated M1/T1 variants (M1/_, T1/_, M1/T1, and_/_). A multivariate analysis through a logistic regression model was performed to identify possible clinical and sociodemographic predictors of higher levels of MDA and TEAC. Variables included in the initial model were gender, age, presence and type of IPI, lifetime history of psychiatric treatment, family history of epilepsy and PD, presence and type of PD, epilepsy duration, age at epilepsy onset, number and types of antiepileptic drug (AED), and laterality of MTS. The Kolmogorov-Smirnov test was applied in order to verify the possibility of normal distribution of residuals, and the Breusch-Pagan test was applied to verify the possibility of homoscedasticity on the model. In addition, analyses of colinearity and multicolinearity matrices between variables were performed. The odds ratio (OR) and $\beta$ coefficient were calculated for risk factors. The 
Stats Direct and GraphPad3 Programs were used. A P value $<0.05$ was considered significant.

\section{Results}

Data from 46 patients ( 25 males; $54.3 \%$ ) and 112 controls ( 62 males; $55.3 \%)(P=0.23)$ were included. The mean age of patients and controls were of $43.6 \pm 1.3$ and $42.7 \pm 3.2$, respectively $(P=0.54)$. Patients' group presented more history of previous psychiatric treatment $(\mathrm{P}=$ 0.001 ), family history of epilepsy ( $P=0.002$ ), and family history of PD $(P=0.03)$. Comparisons between group with TLE-MTS and control group are showed in Table 1.

In group with TLE-MTS, the mean duration of epilepsy and mean age at epilepsy onset were $25.1 \pm 11.3$ and $13.4 \pm 2.2$ years, respectively. The MTS occurred more frequently on the left side (31 patients; $67.4 \%$ ). Eleven patients (23.9\%) had a history of IPI, with febrile seizures as the most frequent (six cases; 54.5\%). There were also four cases (36.3\%) of meningoencephalitis and one case $(9.2 \%)$ of perinatal hypoxia. Forty-four patients (95.6\%) had used at least two or more AEDs at the moment of the study. Carbamazepine (CBZ) was the most frequent $A E D$, prescribed to 31 patients (67.4\%), followed by phenytoin (PHT) (20 patients; 43.4\%), phenobarbital (PB) (14 patients; 30.4\%), topiramate (TPM) (10 patients; 21.7\%), and oxcarbazepine (OXC) (7 patients; 15.2\%). Benzodiazepines (BZD), particularly clobazam (CLB), were the most common adjunctive drugs, prescribed to 25 patients (54.3\%). Patients with PD presented a significantly longer epilepsy duration $(P=0.03)$, younger age at epilepsy onset $(P=$ $0.03)$, and more history of previous psychiatric treatment $(\mathrm{P}<0.001)$ than patients without PD. These data are summarized in Table 2.

Twenty-seven patients with TLE-MTS presented PD (58.6\%). Major depressive disorder (MDD) was the most frequent PD diagnosed (15 patients; $32.6 \%$ ). Nine patients (19.5\%) presented with anxiety disorders. Among them, seven patients had generalized anxiety disorder (GAD), and two patients had social phobia. Psychotic disorders were observed in seven patients (15.2\%): IIP in four patients (8.6\%) and PIP in three patients (6.5\%). Somatoform disorders were observed in five $(10.8 \%)$ patients, and all of them had psychogenic nonepileptic seizures (PNES). Nine patients fulfilled criteria for two PD. Among patients taking psychiatric drugs, sertraline was the most frequent antidepressant drug, prescribed to 13 patients, followed by fluoxetine (seven). Risperidone was the most common antipsychotic (four patients), followed by olanzapine (three). Among the 112 individuals of the control group, there were 15 individuals with PD (13.4\%). The most prevalent were GAD (eight cases; 7.1\%) and MDD (five cases; $4.4 \%)$. There were also two cases of alcohol abuse (1.8\%). Patients with TLE-MTS presented significantly more cases of MDD $(\mathrm{P}<0.001)$, anxiety disorders $(P<0.001)$, psychoses $(P<0.001)$ and of somatoform disorders $(\mathrm{P}<0.001)$. The number and types of $\mathrm{PD}$ in both groups are described in Fig. 1.

Regarding the serum level analyses of MDA and TEAC, significantly higher levels of both substances in group with TLE-MTS were observed. The mean value of MDA was of $201 \mathrm{ng} / \mathrm{ml}$ in patients with TLE-MTS and

Table 1

Clinical and demographic data of patients with TLE-MTS compared with a control group.

\begin{tabular}{llll}
\hline Clinical/demographic data & Patients & Control group & P \\
\hline Number of patients (\%) & 46 & 112 & - \\
Gender - males (\%) & $25(54.3)$ & $62(55.3)$ & $0.23^{\mathrm{a}}$ \\
Age (mean \pm SD) & $43.6 \pm 1.3$ & $42.7 \pm 3.2$ & $0.54^{\mathrm{b}}$ \\
Years of education (mean \pm SD) & $7.4 \pm 2.6$ & $9.2 \pm 3.5$ & $0.62^{\mathrm{b}}$ \\
Previous psychiatric treatment (\%) & $9(19.5)$ & $5(4.1)$ & $0.001^{*, \mathrm{c}}$ \\
Family history of epilepsy (\%) & $17(26.0)$ & $11(9.8)$ & $0.002^{*, \mathrm{a}}$ \\
Family history of psychiatric disorders (\%) & $7(15.2)$ & $8(7.1)$ & $0.03^{*, \mathrm{c}}$ \\
\hline * P<0.05. & & & \\
a Chi-square test. & & & \\
b Student's $t$-test. & & & \\
c Fisher's exact test. & & & \\
\end{tabular}

Table 2

Clinical and demographic data of patients with TLE-MTS with and without psychiatric disorders.

\begin{tabular}{llll}
\hline Clinical/demographic data & $\begin{array}{l}\text { Patients } \\
\text { with PD }\end{array}$ & $\begin{array}{l}\text { Patients } \\
\text { without PD }\end{array}$ & P \\
\hline Number of patients (\%) & 27 & 19 & - \\
Gender - males (\%) & $15(55.5)$ & $10(52.6)$ & $0.83^{\mathrm{a}}$ \\
Age at epilepsy onset (mean \pm SD) & $11.2 \pm 3.2$ & $14.1 \pm 2.5$ & $0.03^{*, \mathrm{~b}}$ \\
Years of epilepsy duration (mean \pm SD) & $22.1 \pm 10.4$ & $25.7 \pm 12.0$ & $0.03^{*, \mathrm{~b}}$ \\
Previous psychiatric treatment (\%) & $8(29.6)$ & $1(5.2)$ & $<0.001^{*, \mathrm{c}}$ \\
Family history of epilepsy (\%) & $10(37.0)$ & $7(36.8)$ & $0.82^{\mathrm{a}}$ \\
Family history of psychiatric disorders (\%) & $5(18.5)$ & $2(10.5)$ & $0.54^{\mathrm{c}}$ \\
MTS lateralization & $18(66.6)$ & $13(68.4)$ & $0.83^{\mathrm{a}}$ \\
Left & $9(33.4)$ & $6(31.6)$ & $0.87^{\mathrm{a}}$ \\
Right & & & \\
Most frequently used AEDs (\%) & $20(74.0)$ & $11(57.8)$ & $0.23^{\mathrm{a}}$ \\
Carbamazepine & $12(44.4)$ & $8(42.1)$ & $0.78^{\mathrm{a}}$ \\
Phenytoin & $9(33.3)$ & $5(26.3)$ & $0.64^{\mathrm{a}}$ \\
Phenobarbital & $6(22.2)$ & $4(21.0)$ & $0.74^{\mathrm{a}}$ \\
Topiramate & $5(18.5)$ & $2(10.5)$ & $0.54^{\mathrm{c}}$ \\
Oxcarbazepine & $15(55.5)$ & $10(52.6)$ & $0.83^{\mathrm{a}}$ \\
Benzodiazepines & & & \\
Presence and type of IPI (\%) & $20(74.0)$ & $14(73.6)$ & $0.92^{\mathrm{a}}$ \\
No IPI & 1 & 0 & - \\
Perinatal hypoxia & $5(18.5)$ & $3(15.7)$ & $0.88^{\mathrm{a}}$ \\
Febrile seizures & $2(7.4)$ & $2(10.5)$ & $0.62^{\mathrm{a}}$ \\
Meningoencephalitis & & & \\
\hline
\end{tabular}

AED: antiepileptic drug; MTS: mesial temporal sclerosis; SD: standard deviation.

* $\mathrm{P}<0.05$.

a Chi-square test.

b Student's t-test.

c Fisher's exact test.

of $137.4 \mathrm{ng} / \mathrm{ml}$ in controls $(\mathrm{P}<0.0001)$, whereas, TEAC values were of $2.87 \mathrm{nmol} / \mathrm{L}$ in patients with TLE-MTS and of $2.60 \mathrm{nmol} / \mathrm{L}$ in controls $(\mathrm{P}<0.0001)$. When serum levels of MDA and TEAC of patients with $\mathrm{PD}$ were compared with patients without PD, no significant differences were observed. However, when patients with TLE-MTS with MDD were compared with patients with PD (except MDD), to patients without PD and to control groups with or without PD, significant differences were observed on MDA serum levels $(\mathrm{P}=0.002 ; \mathrm{P}=0.003$ and $\mathrm{P}<0.001$, respectively). The same findings were observed when TEAC values of patients with TLE-MTS with MDD were compared with patients with PD (except MDD), to patients without PD and to different compositions of control group, significant differences were observed on MDA serum levels $(\mathrm{P}=0.003 ; \mathrm{P}=0.02$ and $\mathrm{P}<0.001$, respectively). These data are properly showed in Figs. 2 and 3.

The final adjusted model (sensitivity $72.7 \%$; specificity $85.5 \%$; positive predictive value $65.5 \%$; negative predictive value $78.3 \%$; area under the curve 0.798 ) on multivariate logistic regression analysis observed that presence of $\mathrm{MDD}(\mathrm{OR}=4.32 ; \mathrm{P}=0.008)$, lifetime history of psychiatric treatment $(\mathrm{OR}=3.18 ; \mathrm{P}=0.01)$ and epilepsy duration $(\mathrm{OR}=3.39 ; \mathrm{P}=0.009)$ appeared as significant risk factors for higher levels of MDA (Table 3). The other final adjusted model (sensitivity $71.5 \%$; specificity $83.2 \%$; positive predictive value $64.5 \%$; negative predictive value $76.5 \%$; area under the curve 0.756 ) on multivariate logistic regression analysis observed that the presence of $\mathrm{MDD}(\mathrm{OR}=3.55 ; \mathrm{P}=$ $0.008)$ and epilepsy duration $(\mathrm{OR}=3.27 ; \mathrm{P}=0.01)$ appeared as significant risk factors for higher levels of TEAC (Table 4).The result on Kolmogorov-Smirnov test $(0.162 ; \alpha=0.05)$ confirmed the normal distribution of residuals, and the result on Breusch-Pagan test (0.433; $\alpha=0.05$ ) did not reject the possibility of homoscedasticity in both models. In addition, colinearity and multicolinearity matrices did not observe any strong correlation of two or more variables on both analyses.

The analysis of GSTM1 and GSTT1 polymorphisms observed that the null genotypes (absence of at least one of the polymorphisms) showed similar distribution between patients (33\% and 26\%) and controls (45\% and $23 \%)(\mathrm{P}=0.22$ and $\mathrm{P}=0.85$, respectively). The presence of $\mathrm{M} 1 / \mathrm{T} 1$ 


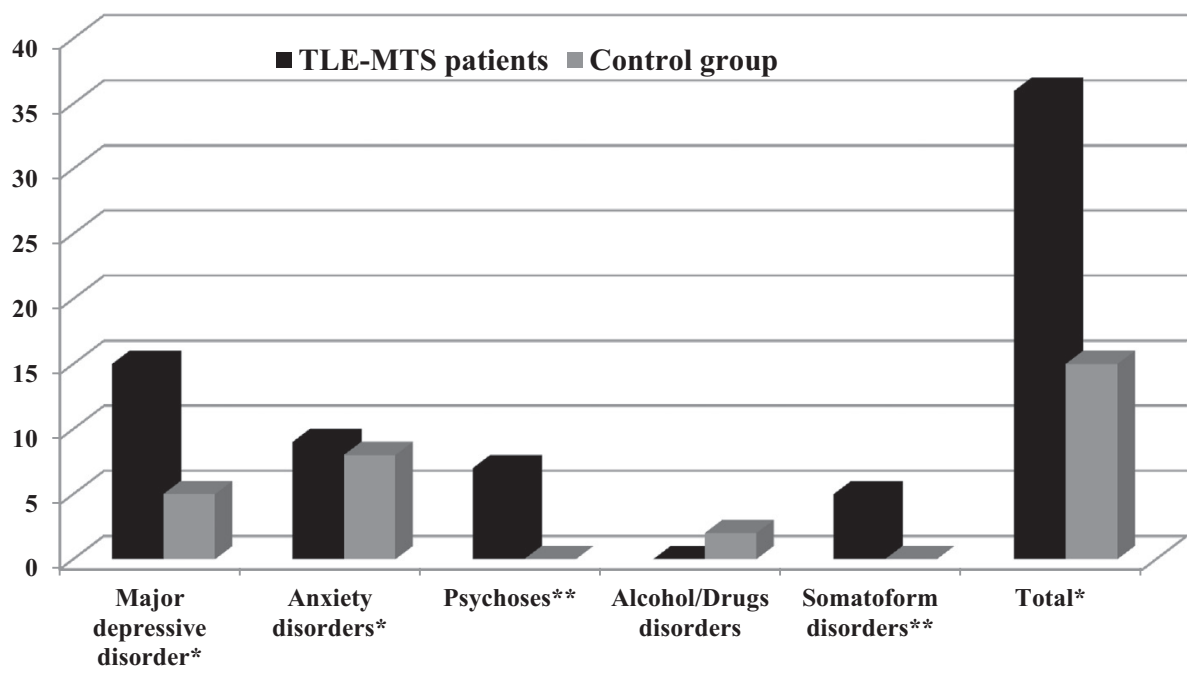

Fig. 1. Psychiatric disorders in patients with TLE-MTS and in a control group. ${ }^{*} \mathrm{P}<0.05$ (Chi-square); ${ }^{* *} \mathrm{P}<0.05$ (Fisher's exact test).

was observed with higher prevalence in both groups (63\% and $43 \%$ ), but without significant differences $(P=0.80)$.

\section{Discussion}

The aim of the present controlled study was to verify the serum levels of MDA and TEAC, biomarkers of OS, and the genetic polymorphisms of GSTs in a series of patients with refractory TLE-MTS compared with a healthy control group. Higher serum levels of MDA and of TEAC were observed in patients with refractory TLE-MTS in comparison with the control group, which were even higher among patients with TLE-MTS with MDD.

Studies have shown that OS could be associated with neurodegenerative disorders, such as Alzheimer's and Parkinson's diseases, pathologies involving chronic inflammations with consequent higher levels of OS due to an increase of ROS and RNS production [23-26]. In this context, OS markers have received substantial attention in recent years and many of them have been studied, such as MDA and TEAC [27]. In the same way, a growing number of studies have suggested an important role of OS in pathophysiology of several PD and particularly in MDD [28-31], suggesting that MDD could be accompanied by a dysregulation of antioxidant mechanisms, including altered antioxidant

\section{$\operatorname{MDA}(\mathbf{n g} / \mathrm{ml})$}

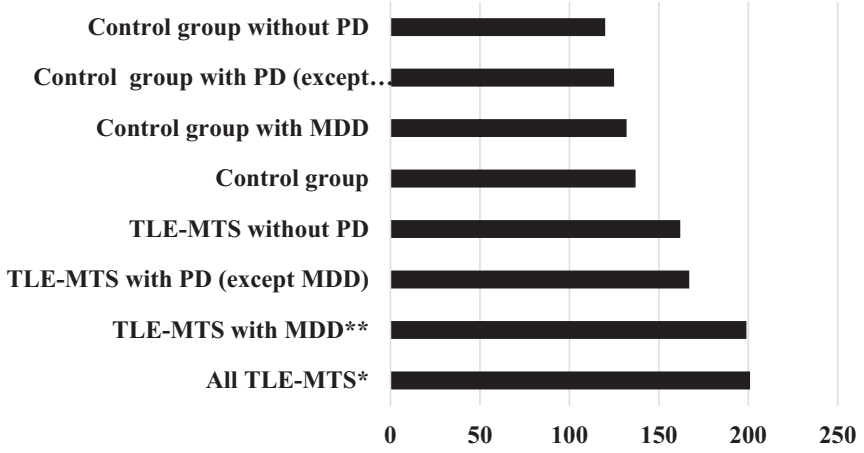

Fig. 2. Serum levels of MDA in patients with TLE-MTS and controls. MDA: malondialdehyde; MDD: major depressive disorder; PD: psychiatric disorders; TLE-MTS: temporal lobe epilepsy with mesial temporal sclerosis. ${ }^{*} \mathrm{P}<0.05$ (Analysis of Variance ANOVA - with Bonferroni as post hoc) compared with all control groups. ${ }^{* *} \mathrm{P}<0.05$ (Analysis of Variance - ANOVA - with Bonferroni as post hoc) compared with group with TLE-MTS without PD, group with TLE-MTS with PD (except MDD), and all control groups. capacity and increased FRs [28-30]. Therefore, increased levels of OS markers such as MDA, superoxide dismutase (SOD), and catalase (CAT), could be considered as early markers of depressive episodes in patients with MDD [30]. In addition, studies have observed an improvement of OS markers' levels after the introduction of an antidepressant, reinforcing the hypothesis that the antidepressant activity could be mediated through the improvement of the antioxidant function [29].

The available literature supports a $20-40 \%$ prevalence of psychiatric comorbidity among patients with epilepsy, and mood (20-60\%), anxiety (17-24\%), and psychotic disorders (2-9\%) have been cited as the most common [32-34]. Studies have observed an even higher prevalence of PD in patients with refractory forms of epilepsy, such as refractory TLE-MTS, in which such prevalence can be as high as $70 \%$ [5,35-38]. Previous studies have reported that PD and a lifetime history of MDD predict a worse psychiatric and seizure outcome among patients with refractory TLE-MTS. The most important hypothesis explains this observation by positing that MDD may be a marker of more diffuse cerebral disease, worsening their clinical and psychiatric outcomes [36,39]. In accordance with this hypothesis, a study which aimed to find clinical and sociodemographic predictors of a worse seizure outcome 1 year after surgery in patients with refractory TLEMTS observed that the presence of any presurgical PD was associated

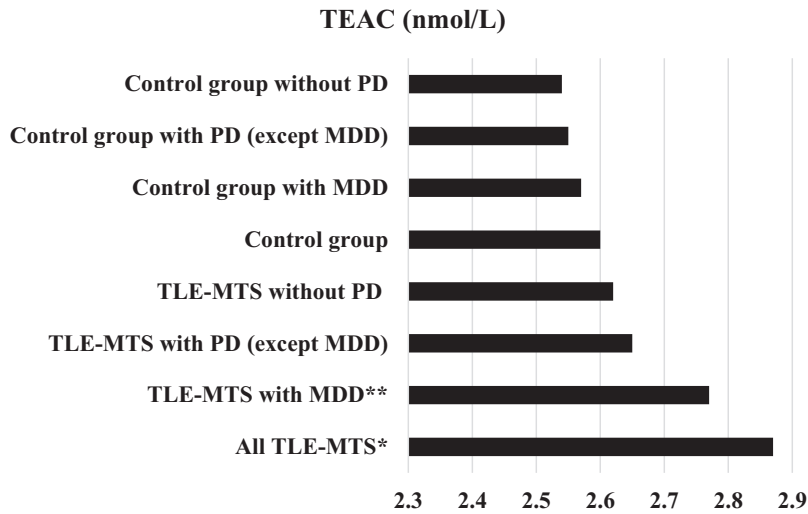

Fig. 3. Serum levels of TEAC in patients with TLE-MTS and controls. MDD: major depressive disorder; PD: psychiatric disorders; TEAC: antioxidant capacity equivalent to the trolox; TLE-MTS: temporal lobe epilepsy with mesial temporal sclerosis. ${ }^{*} \mathrm{P}<0.05$ (Analysis of Variance - ANOVA - with Bonferroni as post hoc) compared with all control groups. ${ }^{* *} \mathrm{P}<0.05$ (Analysis of Variance - ANOVA - with Bonferroni as post hoc) compared with group with TLE-MTS without PD, group with TLE-MTS with PD (except MDD), and all control groups. 
Table 3

Risk factors associated with higher levels of MDA in patients with refractory temporal lobe epilepsy with mesial temporal sclerosis: final adjusted results of a logistic regression model.

\begin{tabular}{llll}
\hline Risk factors & Adjusted OR & CI 95\% & P \\
\hline Age & 1.08 & $0.97-2.27$ & 0.49 \\
Presence of MDD & 3.32 & $1.17-6.45$ & $0.008^{*}$ \\
Age of epilepsy onset & 1.71 & $0.92-2.56$ & 0.26 \\
Epilepsy duration & 3.39 & $1.98-5.13$ & $0.009^{*}$ \\
Lifetime history of psychiatric treatment & 3.18 & $2.72-5.32$ & $0.01^{*}$ \\
Number of AED used & 1.55 & $0.89-3.01$ & 0.45 \\
\hline
\end{tabular}

AED: antiepileptic drug; $\mathrm{CI}$ : confidence interval; MDD: major depressive disorder; OR: odds ratio.

* $\mathrm{P}<0.05$.

with a higher risk for a worse outcome. However, when psychiatric diagnoses were analyzed separately, only the diagnosis of MDD persisted as statistically significant, whereas, the other PD combined (except MDD) were not significant [38]. A recent study with a larger number of patients with TLE submitted to surgery, however, observed that psychiatric comorbidities as a group, and not only MDD, were negatively associated with postoperative seizure-freedom rates. Nevertheless, this study also supported the argument that there are common pathogenetic mechanisms underlying both PD and epilepsy [40]. In the present study, higher levels of OS markers were associated with MDD, epilepsy duration and lifetime history of psychiatric treatment on logistic regression, reinforcing previous results, and the hypothesis of possible negative impacts of MDD on TLE-MTS.

Studies have already observed differences of some markers of OS, such as low levels of antioxidants and high levels of lipid peroxidation, in blood and neuronal tissue of patients with epilepsy [41,42]. Confirming these previous findings, the present study observed higher levels of plasma MDA and TEAC in patients with TLE-MTS compared with controls. Such differences could be due to the higher levels of OS associated with the pathophysiological processes of TLE-MTS [26,43]. However, in other studies that observed such differences of OS markers in patients with epilepsy, authors stated that these phenomena could be partially associated with the effect of AEDs, which could increase the formation of FRs and cause neuronal oxidative damage [44-47].

In the present study, the frequency of nullities of GSTM1 and GSTT1 presented a similar distribution between patients and controls. It is well known that the nullity of GSTM1 and GSTT1, which consist of a deletion of the whole genes, leads to a total absence of the respective enzyme [10]. The homozygous deletion of the GSTM1 gene is observed at frequencies ranging from 20 to $70 \%$ in the different populations, whereas for the GSTT1 gene, this variation is from 11 to $38 \%$ [48] as observed in this study. The null genotype for GSTT1 has been reported to play a protective role in the development of schizophrenia [49-51], while the null genotype for GSTM1 as a risk factor [48,50]. In addition, Shang et al. correlated increased levels of GSTs in endothelial and glial cells with difficult to control epilepsy, as well as resistance to AED treatment [52].

\section{Table 4}

Risk factors associated with higher levels of TEAC in patients with refractory temporal lobe epilepsy with mesial temporal sclerosis: final adjusted results of a logistic regression model.

\begin{tabular}{llll}
\hline Risk factors & Adjusted OR & CI 95\% & P \\
\hline Age of epilepsy onset & 1.10 & $0.97-2.27$ & 0.49 \\
Presence of MDD & 3.55 & $1.47-5.45$ & $0.008^{*}$ \\
Presence of an IPI & 1.60 & $0.92-2.86$ & 0.36 \\
Epilepsy duration & 3.27 & $1.98-5.13$ & $0.01^{*}$ \\
Number of AED used & 1.52 & $0.89-2.85$ & 0.55
\end{tabular}

AED: antiepileptic drug; IPI: initial precipitant injury; CI: confidence interval; MDD: major depressive disorder; OR: odds ratio.

* $\mathrm{P}<0.05$.
There are important limitations in the present study, which was based on a transversal analysis of a unique peripheral blood sample on a relatively small number of patients. Although the majority of present studies which analyzed OS markers have the same methodology, a longitudinal analysis with serial samples of peripheral blood could provide higher levels of reliability. In addition, since OS marker levels would be quite unspecific and may vary according to the existence of several pathophysiological processes, the presence of a control group was therefore highly necessary. Furthermore, due to methodological limitations, the specific impact of psychiatric medications or AEDs on the level of MDA and TEAC could not be measured. Despite the comparisons proceeded, it is still difficult to ascertain specifically whether the high serum concentrations of MDA and TEAC would be driven by the TLE-MTS, MDD or both, due to methodological limitations. We could not evaluate the prevalence of other PD not covered by the diagnostic criteria utilized (DSM-5 and ILAE). Finally, although based upon a relatively small number of patients, our findings are concordant with the extant literature and address a pure population of patients with TLE-MTS.

In conclusion, the present cross-sectional study observed significantly higher serum levels of MDA and of TEAC, OS markers that have been frequently studied for several diseases, in patients with refractory TLE-MTS compared with a healthy control group. In addition, patients with TLE-MTS and MDD presented higher levels when compared with patients with TLE-MTS without PD and with another PD except MDD. Therefore, MDD was observed as an important issue associated with OS in refractory TLE-MTS. Further, longitudinal studies with larger number of patients and more patients with PD except MDD are thus necessary to investigate this complex association of OS, refractory TLEMTS, and PD.

\section{Acknowledgments}

This work was supported by the Brazilian National Council for Scientific Research (CNPq: 443742/2014-6) and Medical School of Sao José do Rio Preto, Brazil (FAMERP) (grant 110400).

\section{Conflicts of interest}

The authors confirm that they do not have any financial or personal relationships with other persons or organizations that could inappropriately influence their work. Moreover, they confirm that they have no additional conflicts of interest to disclose.

\section{References}

[1] Puttachary S, Sharma S, Stark S, Thippeswamy T. Seizure-induced oxidative stress in temporal lobe epilepsy. Biomed Res Int 2015;2015:745.613.

[2] Sun Z, Zuo H, Yuan D, Sun Y, Zhang K, Cui Z, et al. Predictors of prognosis in patients with temporal lobe epilepsy after anterior temporal lobectomy. Exp Ther Med 2015; 10:1896-902.

[3] Pack AM. Do patients with refractory temporal lobe epilepsy shift their quality of life priorities after having surgery. Epilepsy Curr 2015;15(1):32-3.

[4] Baker-Haliski M, Sills GJ, White HS. What are the arguments for and against rational therapy for epilepsy. Adv Exp Med Biol 2014;813:295-308.

[5] Araujo-Filho GM, Mazzeto L, Yacubian EMT. Psychiatric and behavioral effects of the antiepileptic drugs and their action as mood stabilizers. J Epilepsy Clin Neurophysiol 2011:17(2):65-9.

[6] Araujo-Filho GM, Furlan AER, Ribeiro AESA, Marques LHN. Psychiatric disorders as "hidden" contraindications for pre-surgical VEEG in patients with refractory epilepsy: a retrospective cohort study in a tertiary center. Epilepsy Behav 2015;45:35-8.

[7] Salim S. Oxidative stress and psychological disorders. Curr Neuropharmacol 2014; 12(2):140-7.

[8] Aguiar CC, Almeida AB, Araújo PV, de Abreu RN, Chaves EM, do Vale OC, et al. Oxidative stress and epilepsy: literature review. Oxid Med Cell Longev 2012;2012:795259. https://doi.org/10.1155/2012/795259.

[9] Socha MJ, Boerman EM, Behringer EJ, Shaw RL, Domeier TL, Segal SS. Advanced age protects microvascular endothelium from aberrant $\mathrm{Ca}_{2}{ }^{+}$influx and cell death induced by hydrogen peroxide. J Physiol 2015:593(9):2155-69.

[10] Chbili C, B'chir F, Fredj MB, Saguem BN, Amor SB, Ammou SB, et al. Effects of glutathione $S$-transferase M1 and T1 deletions on epilepsy risk among a Tunisian population. Epilepsy Res 2014;108(7):1168-73. 
[11] Kim SK, Kang SW, Chung JH, Parque HJ, Cho KB, Parque MS. Genetic polymorphisms of glutathione-related enzymes (GSTM1, GSTT1, and GSTP1) and schizophrenia risk: a meta-analysis. Int J Mol Sci 2015;16(8):19602-11.

[12] Atamer A, Kurdas-Ovunc AO, Yesil A, Atamer Y. Evaluation of paraoxonase, malondialdehyde, and lipoprotein levels in patients with asymptomatic cholelithiasis. Saudi J Gastroenterol 2014;20(1):66-73.

[13] Gilca M, Piriu G, Gaman L, Delia C, Iosif L, Atanasiu V, et al. A study of antioxidant activity in patients with schizophrenia taking atypical antipsychotics. Psychopharmacology (Berl) 2014;231(24):4703-10.

[14] Commission on Classification and Terminology of the International League Against Epilepsy. Proposal for revised classification of epilepsies and epileptic syndromes. Epilepsia 1989;30:389-99.

[15] American Psychiatric Association. Diagnostic and statistical manual for mental disorders DSM - IV (text revision)4th ed. ; 2000[Washington].

[16] Krishnamoorthy ES, Trimble MR, Blumer D. The classification of neuropsychiatric disorders in epilepsy: a proposal by the ILAE commission on psychobiology of epilepsy. Epilepsy Behav 2007;10:349-53.

[17] Salazar LA, Hirata MH, Cavalli SA, Machado MO, Hirata RD. Optimized procedure for DNA isolation from fresh and cryopreserved clotted human blood useful in clinical molecular testing. Clin Chem 1998;44:1748-50.

[18] Kadioglu E, Taçoy G, Özçağlı E, Okyay K, Akboğa MK, Çengel A, et al. The role of oxidative DNA damage and GSTM1, GSTT1, and hOGG1 gene polymorphisms in coronary artery disease risk. Anatol J Cardiol 2016. https://doi.org/10.14744/ AnatolJCardiol.2016.6697.

[19] Almeida EA, Marques SA, Klitzke CF, Bainy ACD, Medeiros MHG, Di Mascio P, et al. DNA damage in digestive gland and mantle tissue of the mussel Perna perna. Comp Biochem Physiol 2003;135:295-303.

[20] Almeida EA, Miyamoto S, Bainy AC, Medeiros MH, Di Mascio P. Protective effect of phospholipid hydroperoxide glutathione peroxidase (PHGPX) against lipid peroxidation in mussels Perna perna exposed to different metals. Mar Pollut Bull 2004; 49:386-92.

[21] Miller NJ, Rice-Evans C, Davies MJ, Gopinathan V, Milner A. A novel method for measuring antioxidant capacity and its application to monitoring the antioxidant status in premature neonates. Clin Sci 1993;84(4):407.

[22] Re R, Pellegrini N, Proteggente A, Pannala A, Yang M, Rice-Evans C. Antioxidant activity applying an improved ABTS radical cation decolorization assay. Free Radic Biol Med 1999;26:1231-7.

[23] Chang SJ, Yu BC. Mitochondrial matters of the brain: mitochondrial dysfunction and oxidative status in epilepsy. J Bioenerg Biomembr 2010;42(6):457-9.

[24] Dönmezdil N, Çevik MU, Özdemir HH, Tasin M. Investigation of PON1 activity and MDA levels in patients with epilepsy not receiving antiepileptic treatment. Neuropsychiatr Dis Treat 2016;12:1013-7.

[25] Ramírez A, Vázquez-Sánchez AY, Carrión-Robalino N, Camacho J. Ion channels and oxidative stress as a potential link for the diagnosis or treatment of liver diseases. Oxid Med Cell Longev 2015;16:01-17.

[26] Mehvari J, Motlagh FG, Najafi M, Ghazvini MR, Naeini AA, Zare M. Effects of vitamin E on seizure frequency, electroencephalogram findings, and oxidative stress status of refractory epileptic patients. Adv Biomed Res 2016;5:36.

[27] Peker E, Oktar S, Ari M, Kozan R, Doğan M, Cağan E, et al. Nitric oxide, lipid peroxidation, and antioxidant enzyme levels in epileptic children using valproic acid. Brain Res 2009;1297:194-7.

[28] Tsai MC, Huang TL. Increased activities of both superoxide dismutase and catalase were indicators of acute depressive episodes in patients with major depressive disorder. Psychiatry Res 2016;235:38-42.

[29] Jiménez-Fernández S, Gurpegui M, Diaz-Atienza F, Pérez-Costillas L, Gerstenberg M, Correl CU. Oxidative stress and antioxidant parameters in patients with major depressive disorder compared to healthy controls before and after antidepressant treatment: results from a meta-analysis. J Clin Psychiatry 2015;76:1658-67.

[30] Liu T, Zhong S, Liao X, Chen G, He T, Lai S, et al. A meta-analysis of oxidative stress markers in depression. PLoS One 2015;10(10):e0138904. https://doi.org/10.1371/ journal.pone.0138904.

[31] Bajpai A, Verma AK, Srivastava M, Srivastava R. Oxidative stress and major depression. J Clin Diagn Res 2014;8(12):CC04-7. https://doi.org/10.7860/JCDR/2014/ 10258.5292.
[32] Gaitatzis A, Trimble MR, Sander JW. The psychiatric comorbidity of epilepsy. Acta Neurol Scand 2004;110:207-20.

[33] Devinsky O. Psychiatric comorbidity in patients with epilepsy: implications for diagnosis and treatment. Epilepsy Behav 2003;4:2-10.

[34] Tellez-Zenteno JF, Wiebe S. Prevalence of psychiatric disorders in patients with epilepsy: what we think we know and what we know. In: Kanner AM, Schachter S, editors. Psychiatric controversies in epilepsy. San Diego: Academic Press; 2008. p. 1-18.

[35] Koch-Stoecker S. Psychiatric effects of surgery for temporal lobe epilepsy. In: Trimble M, Schmitz B, editors. The neuropsychiatry of epilepsy. Cambridge: Cambridge University Press; 2002. p. 266-82.

[36] Kanner AM. Depression in epilepsy: prevalence, clinical semiology, pathogenic mechanisms and treatment. Biol Psychiatry 2003;54:388-98.

[37] De Araujo Filho GM, Mazetto L, Macedo JS, Caboclo LO, Yacubian EMT. Psychiatric comorbidity in patients with two prototypes of focal versus generalized epilepsy syndromes. Seizure 2011;20:383-6.

[38] De Araujo Filho GM, Gomes FL, Mazetto L, Marinho MM, Tavares IM, Caboclo LO, et al. Major depressive disorder as a predictor of a worse seizure outcome one year after surgery in patients with temporal lobe epilepsy and mesial temporal sclerosis. Seizure 2012;21:619-23.

[39] Kanner AM, Byrne R, Smith MC, Balabanov AJ, Frey M. Does a lifetime history of depression predict a worse postsurgical seizure outcome following a temporal lobectomy? Ann Neurol 2006;10:19.

[40] Koch-Stoecker SC, Bien CG, Schulz R, May TW. Psychiatric lifetime diagnoses are associated with a reduced chance of seizure freedom after temporal lobe surgery. Epilepsia 2017;58(6):983-93.

[41] Ho YH, Lin YT, Wu CWJ, Chao YM, Chang AYW, Chan JYH. Peripheral inflammation increases seizure susceptibility via the induction of neuroinflammation and oxidative stress in the hippocampus. J Biomed Sci 2015;46(22):01-14.

[42] Pecorelli A, Natrella F, Belmonte G, Miracco C, Cervellati F, Ciccoli L, et al. NADPH oxidase activation and 4-hydroxy-2-nonenal/aquaporin-4 2 adducts as possible new players in oxidative neuronal damage 3 presents in drug-resistant epilepsy. Biochim Biophys Acta 2015;1852(3):507-19.

[43] Menon B, Ramalingam K, Kumar RV. Low plasma antioxidant status in patients with epilepsy and the role of antiepileptic drugs on oxidative stress. Ann Indian Acad Neurol 2014;17(4):398-404.

[44] Yiiksel A, Cengiz M, Seven M, Ulutin T. Erythrocyte glutathione, glutathione peroxidase, superoxide dismutase and serum lipid peroxidation in epileptic children with valproate and carbamazepine monotherapy. J Basic Clin Physiol Pharmacol 2000;11(1):73-81.

[45] Yüksel A, Cengiz M, Seven M, Ulutin T. Changes in the antioxidant system in epileptic children receiving antiepileptic drugs: two-year prospective studies. J Child Neurol 2001;16(8):603-6.

[46] Çevik MU, Varol S, Yücel Y, Altun Y. Serum paraoxonase-1 activities and malondialdehyde levels in patients with epilepsy. Dicle Med J 2012;39(4):557-60.

[47] Nazıroglu M, Yürekli VA. Effects of antiepileptic drugs on antioxidant and oxidant molecular pathways: focus on trace elements. Cell Mol Neurobiol 2013;33(5): 589-99.

[48] Bid HK, Konwar R, Saxena M, Chaudhari P, Agrawal CG, Banerjee M. Association of glutathione $S$-transferase (GSTM1, T1 and P1) gene polymorphisms with type 2 diabetes mellitus in north Indian population. J Postgrad Med 2010;56:176-81.

[49] Gravina P, Spoletini I, Masini S, Valentini A, Vanni D, Paladini E, et al. Genetic polymorphisms of glutathione S-transferases GSTM1, GSTT1, GSTP1 and GSTA1 as risk factors for schizophrenia. Psychiatry Res 2011;187:454-6.

[50] Saadat M, Mobayen F, Farrashbandi H. Genetic polymorphism of glutathione $S$-transferase T1: a candidate genetic modifier of individual susceptibility to schizophrenia. Psychiatry Res 2007;153:87-91.

[51] Harada S, Tachikawa H, Kawanishi Y. Glutathione S-transferase M1 gene deletion may be associated with susceptibility to certain forms of schizophrenia. Biochem Biophys 2001;281:267-71.

[52] Shang W, Liu WH, Zhao XH, Sun QJ, Bi JZ, Chi ZF. Expressions of glutathione $S$-transferase alpha, mu, and pi in brains of medically intractable epileptic patients. BMC Neurosci 2008;9:67. 